\title{
Development of Fluorescent thermoresponsive nanoparticles for temperature monitoring on membrane surfaces
}

\author{
S. Santoro ${ }^{\mathrm{a}, \mathrm{b}}$, V. Sebastian ${ }^{\mathrm{a}, \mathrm{c}}$, A. J. Moro ${ }^{\mathrm{b}}$, C. A. M. Portugal ${ }^{\mathrm{b}}$, J. C. Lima ${ }^{\mathrm{b}}$, I. M. Coelhoso ${ }^{\mathrm{b}}$, J. G.
} Crespo $^{\mathrm{b}}$, R. Mallada ${ }^{\mathrm{a}, \mathrm{c}}$

a Institute of Nanoscience of Aragon (INA) and Department of Chemical, Engineering and Environmental Technology, University of Zaragoza, C/ Mariano Esquillor, s/n, I+D+i Building, 50018, Zaragoza, Spain

b LAQV, REQUIMTE, Departamento de Química, Faculdade de Ciências e Tecnologia, Universidade Nova de Lisboa, 2829-516 Caparica, Portugal

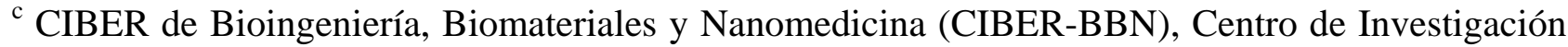
Biomédica en Red. C/ Monforte de Lemos 3-5, Pabellón 11, 28029 Madrid

\begin{abstract}
In this work, tris(phenantroline)ruthenium(II) chloride (Ru(phen) $)_{3}$ ) was immobilized in silica nanoparticles prepared according to the Stöber method. Efforts were devoted on the optimization of the nano-thermometer in terms of size, polydispersity, intensity of the emission and temperature sensitivity. In particular, the immobilization of the luminophore in an external thin shell made of silica grown in a second step on bare silica nanoparticles allowed producing fluorescent monodisperse silica nanoparticles $(420 \pm 20 \mathrm{~nm})$. A systematic study was addressed to maximize the intensity of the emission of the fluorescent nanoparticles by adjusting the concentration of $\mathrm{Ru}$ (phen) ${ }_{3}{ }^{2+}$ in the shell from 0,2 to $24 \mathrm{wt} . \%$, whereas the thickness of the shell is affected by the amount of silica precursor employed.

The luminescent activity of the doped nanoparticles was found to be sensitive to the temperature. In fact, the intensity of the emission linearly decreased by increasing the temperature from $20^{\circ} \mathrm{C}$ to $65^{\circ} \mathrm{C}$. The thermoresponsive nanoparticles were functionalized with long aliphatic chains in order to obtain hydrophobic nanoparticles. The developed nanoparticles were immobilized via dipcoating procedure on the surface of hydrophobic porous membranes, such as Polyvinylidene fluoride (PVDF) prepared via Non-Solvent Induced Phase Separation (NIPS), providing local information about the membrane surface temperature.
\end{abstract}




\section{Introduction}

Temperature is a key parameter affecting mostly all the chemical processes, included membrane processes. Nowadays, thermocouples are commonly used as accurate and inexpensive technologies with a rapid response time for temperature detection. However, the miniaturization of the sensors is the most critical issue of these technologies. In fact, monitoring the temperature with a high spatial resolution at micron and submicron scale is typically demanded in innovative research fields such as nanomedicine, biotechnology and microfluidics [1-5].

In the recent years, luminescent probes have been considered a powerful tool for the monitoring of the temperature at nanoscale, since the properties of their emissions (fluorescence or phosphorescence) are generally affected by the temperature [6-8]. Luminescent probes have been studied as a promising alternative since their immobilization in nanoparticles increases their optical properties (i.e. photostability, emission quantum yield, isolation from possible quenchers) [9-10]. Luminescent probes silica-doped nanoparticles have been considered ideal for monitoring biochemical processes, since they are chemically inert, biocompatible, not subjected to microbial attack and with easy tailoring [11-12]. Several luminescent probes such as rhodamine 6G, acridine orange, $\mathrm{Ru}(\mathrm{bpy})_{3}$ and $\mathrm{Ru}(\mathrm{phen})_{3}$ [13-17], were successfully immobilized on silica nanoparticles for the development of nano-thermometers. In fact, Ruthenium(II) polypyridyl complexes, in particular $\mathrm{Ru}$ (phen $)_{3}$ and $\mathrm{Ru}(\mathrm{byp})_{3}$, are widely explored as luminescent probes for temperature detection because of the strong temperature dependence of their photochemical properties [18]. The thermal quenching of the emission is attributed to the thermal-driven non-radiative decay of the excited state, which leads to a decrease of the intensity of the emission and life time of the excited state, described by an Arrhenius-type model, approximated to a linear relationship at low temperature [19].

Temperature sensitive paints (TSP) based on the dispersion of emissive doped nanobeads or nanoparticles were successfully employed for two dimension temperature mapping in aerodynamics, providing information on aerodynamic performance and on heat transfer in structures [20-21]. This technology could be of interest for the development of smart coatings for monitoring the temperature on membrane surfaces. Temperature plays a key role in membrane processes, in particular in those where the driving force is guaranteed by gradient of temperature across the membrane, such as direct contact membrane distillation. In this case, the heat transfer between the boundary layer of streams and membrane surface produces a temperature profile along the membrane surface (thermal polarization) which dramatically affects the global performance of the process [22-23]. Efforts towards a better understanding of this phenomenon have been performed through the development of modelling studies. However, these studies have been limited 
by the lack of experimental data, since the non-invasive monitoring of temperature at the membrane surface with high enough spatial resolution conditions is a challenging task still not solved. Ali et al. studied the effect of various hydrodynamic and thermal conditions on heat and mass transport in direct contact membrane distillation using a cell containing different thermocouples [24][24]. Optical techniques present the advantage of a non-invasive monitoring and were employed for evaluation of temperature polarization in spacer-filled by means of Thermochromic Liquid Crystals [25].

The aim of this work is the development of a nano-thermometer for the measurement of the temperature on the surface of a membrane based on the attachment of fluorescent silica nanoparticles on its surface. Two different approaches will be evaluated for the immobilization of $\mathrm{Ru}$ (phen) ${ }_{3}{ }^{2+}$ in silica nanoparticles: i) immobilization of the probe in the silica matrix (Core NPs), ii) immobilization of the probe in an external shell (Shell NPs) grown in a second step on bare silica nanoparticles (Bare NPs). Efforts have been devoted on finding a compromise between the photophysical properties and the nanoparticle morphology, in particular, size and polydispersity. Subsequently, Shell NPs will be functionalized to confer to the nanoparticles an hydrophobic behaviour and then immobilized on membrane surface via dip-coating procedure. The stability of the attached nanoparticles will be studied, as well as, the sensitivity to the temperature of the modified membrane.

In this work, silica nanoparticles doped with a luminescent molecule sensitive towards the temperature are proposed as an innovative technology for non-invasive, on-line, in-situ monitoring of the temperature in different processes. In particular, the membrane community will be benefit from this tool and will be able to validate theoretical models and answer important questions related to heat transfer and temperature polarization in membrane processes.

\section{Experimental section}

\subsection{Materials}

Tetraethoxysilane (TEOS), absolute ethanol (EtOH), ammonium hydroxide (ACS reagent 28\%), Dodecyltriethoxysilane and the luminophore tris(phenantroline)ruthenium(II) chloride $\left(\mathrm{Ru}(\mathrm{phen})_{3}\right.$ ) employed for the preparation of $\mathrm{SiO}_{2} \mathrm{NPs}$ were purchased from Sigma Aldrich (Spain). Hydrophobic porous membranes made of poly (vinylidenefluoride) ((PVDF) Solef® 6012, Solvay Specialty Polymers, Italy) were prepared using the Non-Solvent Induced Phase separation (NIPS) technique . The solvent was N-methylpyrrolidone (NMP, 99\%, Carlo Erba Reagents, Spain),

whereas distilled water and lithium chloride ( $\mathrm{LiCl}$, 98\%, Carlo Erba Reagents, Spain) were employed as additives. The phase inversion step was induced by a coagulation bath containing 
distilled water and 2-propanol (99.9\%, Carlo Erba Reagents, Spain) 50/50 wt./wt. Polydimethylsiloxane (PDMS) elastomer (Sylgard 184, Dow Corning, USA) was used to attach silica nanoparticles on membrane surface.

\subsection{Synthesis of $\mathrm{SiO}_{2} \mathrm{NPS}$}

For the preparation of the tris(phenantroline)ruthenium(II)-doped silica nanoparticles two different immobilization approaches were employed: a) inside of a silica core, b) in a silica shell grown on a bare silica core. The two different methods are summarized in Figure 1.

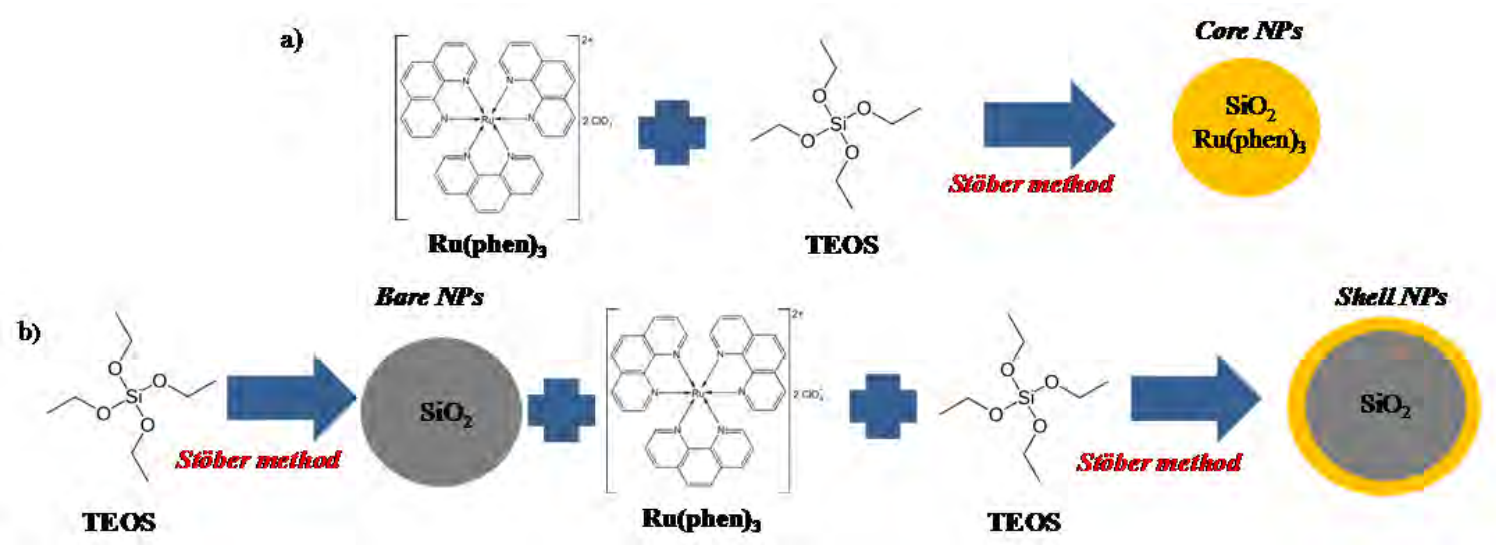

Figure 1: Scheme of preparation of Core and Shell doped silica nanoparticles.

Bare NPs were synthesized following the procedure proposed by Stöber [12], which is based on the hydrolysis and condensation reaction of TEOS in presence of $\mathrm{NH}_{3}$ and $\mathrm{H}_{2} \mathrm{O}$ in EtOH (molar ratio TEOS: EtOH: $\left.\mathrm{NH}_{4} \mathrm{OH}_{\mathrm{aq}}=1: 187: 22\right)$. Briefly, $6 \mathrm{ml}$ of ammonia were added to $43.1 \mathrm{ml}$ of ethanol and stirred for $5 \mathrm{~min}$. Subsequently, $0.91 \mathrm{ml}$ of TEOS were added to the basic solution. The solution was magnetically stirred at $400 \mathrm{rpm}$ for 3 hours at $50^{\circ} \mathrm{C}$. The $\mathrm{SiO}_{2} \mathrm{NPs}$ were collected by centrifugation, washed 3 times with ethanol and re-dispersed in $20 \mathrm{ml}$ of water.

Core NPs were prepared by immobilizing $\mathrm{Ru}(\mathrm{phen})_{3}{ }^{2+}$ in $\mathrm{SiO}_{2}$ during the Stöber reaction. In fact, the method of the preparation is similar to the one employed for the preparation of Bare NPs. However, in this case the $\mathrm{Ru}(\text { phen })_{3}$ was solubilized in the alcoholic solution $(0.04 \mathrm{mg} / \mathrm{ml})$. The concentration of $\mathrm{Ru}(\mathrm{phen})_{3}{ }^{2+}$ with respect to $\mathrm{SiO}_{2}$ is $0.066 \mathrm{~mol} \%(0.73 \mathrm{wt} \%)$.

Shell NPs were prepared by immobilizing the luminescent probe in a shell of silica grown on the surface of bare core $\mathrm{SiO}_{2}$ NPs based on the procedure previously reported by Mirenda et al [26] for tris(bipyridine)ruthenium(II)-doped silica nanoparticles. In this work two parameters have been optimized for the synthesis of Shell NPs: i) the amount of $\mathrm{Ru}(\mathrm{phen})_{3}{ }^{2+}$ fluorophore on the silica shell, that was varied from 0 to $24 \% w t$, in respect to the total silica weight on the shell and ii) the shell size, i.e. amount of TEOS and chemicals for growing the shell (see Table 1) which were 
decreased from the initial (Shell NPs 0.46\%) by a factor of 2 (Shell NPs 0.28\%), 4 (Shell NPs 0.16\%), 10 (Shell NPs 0.069\%) and 20 (Shell NPs 0.036\%), where the value in percentage refers to the weight fraction of the $\mathrm{Ru}(\mathrm{phen})_{3}{ }^{2+}$ load in the NPs made of silica (bare core+shell). In all the cases the weight fraction of fluorophore $\left(\mathrm{Ru}(\mathrm{phen})_{3}{ }^{2+}\right)$ in the silica shell was kept constant at $1.2 \mathrm{wt}$. $\%$.

The procedure for the synthesis is as follows, in the case of Shell NPs $0.46 \%$, (see Table 1), 2.8 micromol of Ru(phen) $)_{3}$ solubilized in $2 \mathrm{ml}$ of EtOH and $0.55 \mathrm{ml}$ of TEOS were added to a solution of $15 \mathrm{ml}$ of bare core $\mathrm{SiO}_{2} \mathrm{NPs}$ in $\mathrm{EtOH}$ (c=5.5 mg ml${ }^{-1}$ ). Then, $1.5 \mathrm{ml}$ of $\mathrm{NH}_{3}(30 \%)$ diluted with $12 \mathrm{ml}$ of $\mathrm{H}_{2} \mathrm{O}$ were added in order to initiate the condensation of TEOS. The solution was stirred for 2 hours at $40^{\circ} \mathrm{C}$ and then kept overnight at room temperature. Finally the Shell NPs were collected by centrifugation, washed with ethanol and dispersed in $15 \mathrm{ml}$ of EtOH. After washing ( 3 times with EtOH), the nanoparticles were re-dispersed in water. In this case, the Shell NPs $0.46 \%$ contain the same amount, $2.8 \mu \mathrm{mol}$ of the luminescent probe as the Core NPs.

Table 1. Preparation of solutions used for the growth of the shell on bare NPs

\begin{tabular}{|c|c|c|c|c|c|}
\hline Shell NPs & $\mathbf{0 . 4 6 0 \%}$ & $\mathbf{0 . 2 8 0 \%}$ & $\mathbf{0 . 1 6 0 \%}$ & $\mathbf{0 . 0 6 9 \%}$ & $\mathbf{0 . 0 3 6 \%}$ \\
\hline EtOH+ Bare NPs (ml) & 15 & 15 & 15 & 15 & 15 \\
\hline $\mathbf{R u}(\mathbf{p h e n})_{3}(\boldsymbol{\mu m o l})$ & 2.80 & 1.40 & 0.70 & 0.28 & 0.14 \\
\hline TEOS (ml) & 0.55 & 0.275 & 0.138 & 0.055 & 0.027 \\
\hline $\mathbf{H}_{2} \mathbf{O}(\mathbf{m l})$ & 12 & 6 & 3 & 1.2 & 0.6 \\
\hline Ammonia (ml) $_{\mathbf{g} \mathbf{S i O}_{2} \text { shell/g BareNPs }}$ & 1.5 & 0.75 & 0.375 & 0.15 & 0.075 \\
\hline
\end{tabular}

\subsection{Immobilization of $\mathrm{SiO}_{2} \mathrm{NPs}$ on PVDF membranes}

PVDF membranes were prepared according to the protocol reported in literature by Drobek et al. [27]. Briefly, PVDF (18wt\%) was slowly added under magnetically vigorous stirring to NMP solution containing $\mathrm{LiCl}(2 \mathrm{wt} \%)$ and $\mathrm{H}_{2} \mathrm{O}(5 \mathrm{wt} \%$ ) as pore former additives. The polymeric solution was stirred overnight at $80^{\circ} \mathrm{C}$ to guarantee the complete dissolution of the polymer and then kept at $80^{\circ} \mathrm{C}$ for 30 min without stirring to remove air bubbles. Finally the dope solution was cast by means of a knife of $0.25 \mathrm{~mm}$ and immersed in the coagulation bath (50wt\% $\mathrm{H}_{2} \mathrm{O}-50 \mathrm{wt} \%$ 2-propanol) for 3 hours ensuring the NIPS process. Finally, the coagulated membranes were washed for 3 hours in a pure water bath to remove all the residual solvents and then were dried for 24 hours at $50^{\circ} \mathrm{C}$. 
In order to turn the hydrophilic character of silica into hydrophobic, Shell NPs were functionalized with Dodecyltriethoxysilane, a TEOS derivative with a long aliphatic chain. $40 \mathrm{ml}$ of Shell NPs dispersed in EtOH were centrifuged to remove the solvent and re-dispersed in toluene (40 $\mathrm{ml})$. The functionalization was carried out at $60^{\circ} \mathrm{C}$ under reflux $\left(\mathrm{N}_{2}\right.$ inert atmosphere): the solution was heated-up to $60^{\circ} \mathrm{C}$ and magnetically stirred, then $1 \mathrm{ml}$ of dodecyltriethoxysilane was injected. After 3 hours, the solution was cooled-down at room temperature and the nanoparticles washed with EtOH three times in order to remove traces of toluene.

Hydrophobic Shell NPs were immobilized on surfaces of PVDF membranes via dip-coating procedure. The dip-coating is a common method for surface modification and consists immersion of the substrate in a sol-gel solution and then withdrawn: gravitational draining and solvent evaporation, accompanied by further densification, result in the deposition of a thin solid layer on the surface of the substrate [28]. On the basis of several parameters, such as the composition and the viscosity of the sol-gel solution, the time of dip-coating is possible to control the structure of the thin coating layer. In this work, the aim of the dip-coating is to immobilize the Shell NPs on PVDF microporous membrane without affecting the chemical-physical properties of the PVDF surface (i.e. contact angle, porosity). There is no specific interaction at the molecular level betwwen the silica nanoparticles containing the probe and the surface of the membrane, then it is expected that the luminescent properties of the prepared nanoparticles will not change. PDMS was employed to favor the adhesion of the NPs on PVDF surfaces and solubilized in toluene at a concentration of $0.4 \mathrm{mg} \mathrm{ml}^{-1}$. After the solubilization of PDMS (3 hours), functionalized Shell $0.46 \% \mathrm{NPs}\left(0.4 \mathrm{mg} \mathrm{ml}{ }^{-}\right.$ ${ }^{1}$ in toluene) were added to the polymeric solution and stirred for 2 hours. Then, the silicon curing agent (wt\% 40.0 - 70.0 Dimethyl, methylhydrogen siloxane) was added (10wt\% with respect to PDMS). Finally, the polymeric solution was sonicated for $30 \mathrm{~min}$. Subsequently, the dip-coating was performed by immersing PVDF membranes for $5 \mathrm{~min}$ in the PDMS solution containing hydrophobic Shell NPs The membranes were then dried for 2 hours at $70^{\circ} \mathrm{C}$, immersed for 5 hours in distilled water to remove non-immobilized NPs and dried once again overnight at room temperature.

\subsection{Characterization}

SEM imaging analyses were acquired in a scanning electron microscope, FEI Quanta FEG250. TEM images were obtained with a transmission electron microscope FEI TECNAI T20, working at $200 \mathrm{kV}$. Particle-size distribution and shell thickness of the resulting nanoparticles during the different synthesis steps were obtained using statistical analysis ( $\mathrm{N}>100$ particles) of SEM images. Results were compared with the NPs size and polydispersity index evaluated by 
Dynamic Light Scattering (DLS) using a Brookhaven 90 Plus instrument (Brookhaven Instruments Corp.). The same equipment was employed for Z-Potential measurements.

Experiments of fluorescence were performed using a LS 55 Fluorescence Spectrometer (Perkin-Elmer) setting the excitation wavelength at $450 \mathrm{~nm}$, excitation and emission slits at $7.5 \mathrm{~nm}$ and a velocity of scanning of $400 \mathrm{~nm} / \mathrm{min}$. For evaluating the sensitivity towards the temperature, $0.5 \mathrm{ml}$ of the prepared $\mathrm{SiO}_{2} \mathrm{NPs}$ were diluted to $10 \mathrm{ml}$, heated-up with a thermostatic bath at different temperatures and, then, 3 samples of $3 \mathrm{ml}$ were transferred into a quartz cuvette, evaluated the real temperature by means of a thermocouple and the intensity of the emission was measured. For each sample 2 cycles were performed .

In the case of the evaluation of the fluorescence of PVDF membranes, the sample was placed in the cuvette on a porous support of glass fixing the angle between the exciting source and the membrane surface at $45^{\circ}$.

The hydrophobicity of the membranes was evaluated by contact angle measurements employing a Dataphysics OCA Series (Neurtex instruments) equipped with $500 \mu \mathrm{L}$ Hamilton syringe. Membrane porosity was determined by gravimetric method following the procedure reported in literature [29]. Briefly, membranes were weighted dry $\left(\mathrm{w}_{\mathrm{d}}\right)$ and wet after the immersion in kerosene for 24 hours $\left(\mathrm{w}_{\mathrm{h}}\right)$. Porosity $(\mathrm{P})$ was calculated according to the following equation:

$$
\mathrm{P}=\frac{\frac{\mathrm{w}_{\mathrm{h}}-\mathrm{w}_{\mathrm{d}}}{\rho_{\mathrm{w}}}}{\frac{\mathrm{w}_{\mathrm{d}}}{\rho_{\mathrm{PVDF}}}+\frac{\mathrm{w}_{\mathrm{h}}-\mathrm{w}_{\mathrm{d}}}{\rho_{\mathrm{w}}}}
$$

where $\rho_{\mathrm{w}}$ is the kerosene density $\left(0.82 \mathrm{~g} \mathrm{~cm}^{-3}\right) \rho_{\mathrm{PVDF}}$ is the PVDF density $\left(1.78 \mathrm{~g} \mathrm{~cm}^{-3}[30]\right)$.

Liquid Entry Pressure (LEP) was determined by placing the membrane in a membrane module filled up with distilled water. The pressure of the compartment was increased 0.2 bar every 5 minutes until water passed through the membrane (LEP).

\section{Results and Discussion}

\subsection{Optimization of fluorescent silica nanoparticles as nanothermomethers}

Bare NPs were prepared using the well-known Stöber method based on the hydrolysis followed by the condensation of TEOS catalyzed by ammonia. According to SEM analysed micrographs (Figure 2), monodispersed Bare NPs with an average diameter of $296 \pm 19 \mathrm{~nm}$ were prepared, as confirmed by DLS measurements $(275 \pm 16 \mathrm{~nm})$ reported in Table 2. Bare NPs showed a narrow size distribution with a polydispersity index of 0.05 . On the other hand; Core NPs 
presented a wide size distribution showing a polydispersity index of 0.26 . Moreover, the average diameter was $555 \pm 90 \mathrm{~nm}$ according to SEM analyses and $544 \pm 88 \mathrm{~nm}$ according to DLS measurements. On the contrary, Shell NPs $0.46 \%$ prepared by coating Bare NPs with an extra layer of $\mathrm{SiO}_{2}$ load with $\mathrm{Ru}\left(\right.$ phen) ${ }_{3}{ }^{2+}$ presented a narrow size distribution (polydispersity index of 0.05 ) with an average diameter of $419 \pm 20 \mathrm{~nm}$ according to SEM picture, coherent with the value of $394 \pm 6$ nm obtained by DLS measurements.

a)

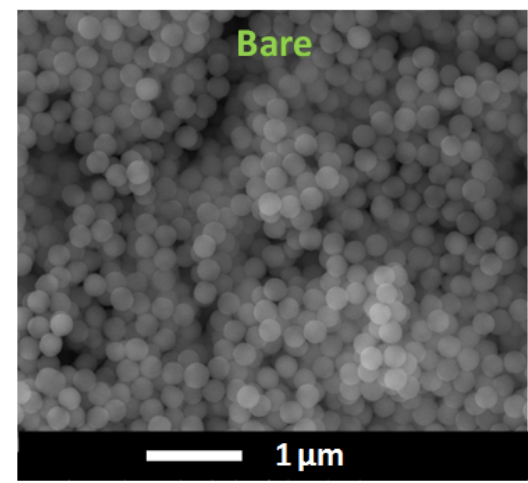

b)

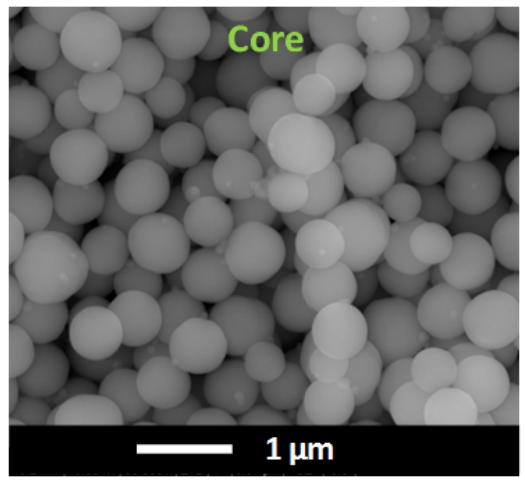

c)

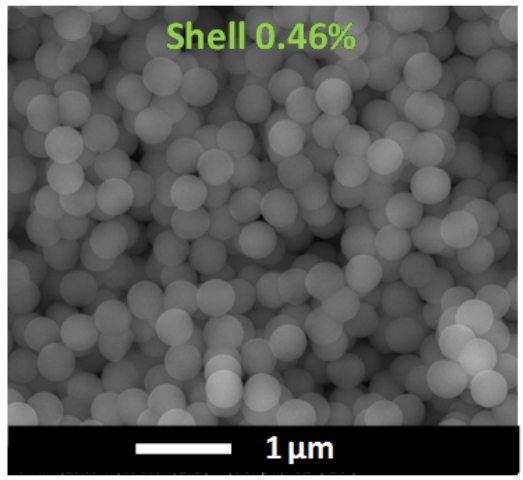

Figure 2. SEM pictures of a)Bare, b) Core and c) Shell NPs $0.46 \%$. The size of the scale bar corresponds to 1 micron in all the images.

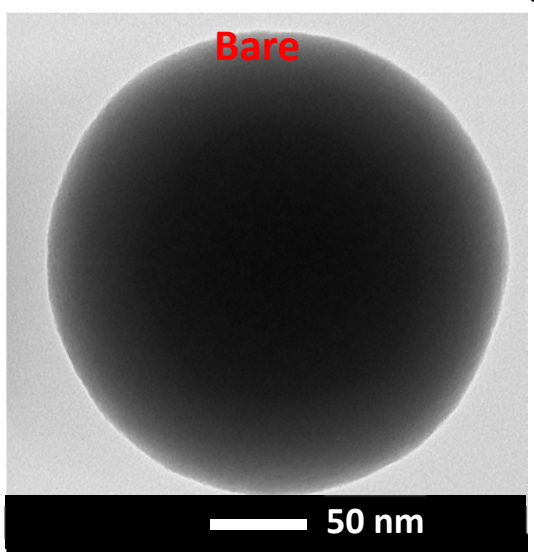

b)

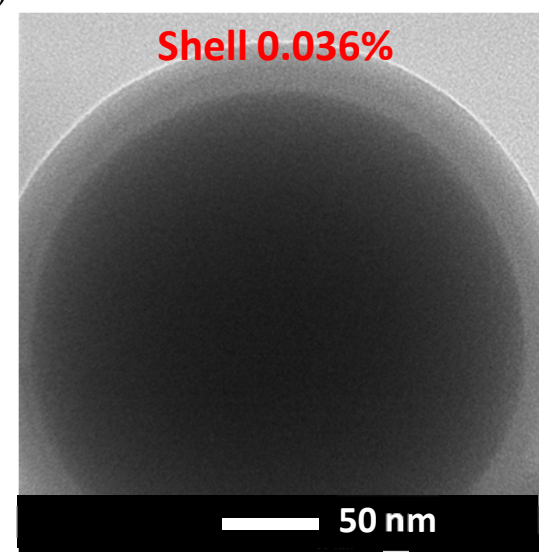

Figure 3. TEM picture of Bare NP (a) and Shell $0.036 \% \mathrm{NP}$. The size of the scale bar corresponds to $50 \mathrm{~nm}$ in all the images.

Table 2. Size and polydispersity index of Bare, Core and Shell NPs.

\begin{tabular}{|c|c|c|c|}
\hline $\mathbf{S i O}_{2} \mathbf{N P s}$ & Diameter (nm) & Diameter (nm) & Polydispersity index \\
& SEM & DLS & DLS \\
\hline Bare & $296 \pm 19$ & $275 \pm 16$ & 0.05 \\
\hline Core & $555 \pm 90$ & $544 \pm 88$ & 0.26 \\
\hline
\end{tabular}




\begin{tabular}{|l|l|l|l|}
\hline Shell 0.46\% & $419 \pm 20$ & $394 \pm 6$ & 0.05 \\
\hline
\end{tabular}

In general, the Stöber protocol generates electrostatically stabilised, spherical and monodisperse particles. This is consequence of the hydrolysis and condensation of silica precursors, such as TEOS, in ethanol solution in the presence of aqueous ammonium hydroxide mixture as a condensation reaction catalyst. Indeed, in the case of Bare NPs homogeneous nucleation forms silica particles of $296 \mathrm{~nm}$ in size. This method is rather simple and it can enable the incorporation of both organic and inorganic markers [31]. However, the immobilization of the probes may lead to a non-uniform particle size [32]. The high polydispersity of Core NPs suggests that the addition of the ruthenium complex hindered the formation of NPs due to the steric effect of phenantroline ligands affecting the hydrolysis and condensation reactions. On the contrary, the $\mathrm{Ru}(\mathrm{phen})_{3}{ }^{2+}$-Shell grew on a well-organized structure as the Bare NPs leading to a narrow size distribution and a low polydispersity index (Figure 2 and Table 2) [33]. The presence of the external Ru(phen) ${ }_{3}{ }^{2+}$-Shell is also confirmed by TEM analyses of the single NPs (Figure 3). In fact, the diameter of the Shell NPs is bigger compared to that one observed for Bare NPs due to the condensation of extra amount of silica. Moreover, under TEM observation conditions at high voltage, the Ru(phen) ${ }_{3}{ }^{2+}$-Shell appeared lighter due to the burning of the organic ligands of the complex of Ruthenium.

In Figure 4a) the excitation and emission spectra of Bare, Core and Shell $0.46 \%$ NPs are reported. Measurements evidenced that Bare NPs are not emissive, as expected, whereas the immobilization of the luminescent probe in silica conferred to NPs luminescent properties. In fact, an evident peak of emission, with a maximum at $590 \mathrm{~nm}$, could be observed by exciting the NPs dispersed in water at $450 \mathrm{~nm}$ (maximum of excitation). Moreover, Shell NPs prepared by the immobilization of the luminescent probe in an external shell made of silica, growth in a second step, presented higher emission with respect to those prepared by the injection of the luminophore at the beginning of the Stöber reaction (Core NPs). In fact, the hydrolysis and condensation of TEOS is fast under the catalysis of the ammonia and the complex of Ruthenium molecules tend to aggregate in the silica core leading to non-emissive aggregates of the luminescent probe by introducing $\mathrm{Ru}(\mathrm{phen})_{3}{ }^{2+}$ at the beginning of the reaction [34]. On the other hand, if the luminophores were added in a second step (Shell NPs), they will be gradually absorbed by polar interaction on the layers of the growing silica shell upon the deposition of the remaining monomers of silica. 
a)

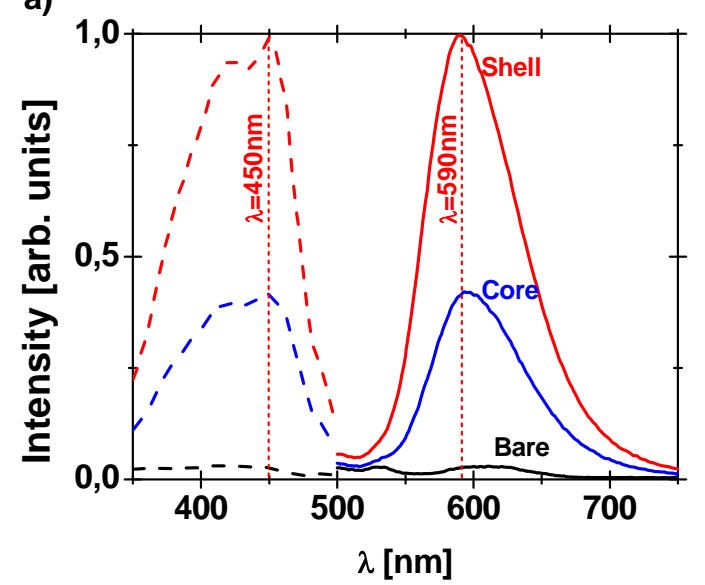

b)

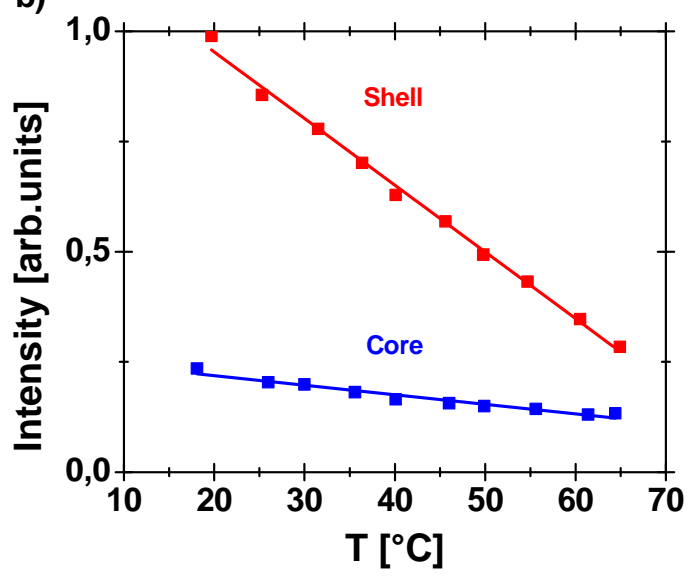

Figure 4. (a) Excitation (dashed line) and emission (solid line) spectra of Bare, Core and Shell 0.46\% NPs a) and (b) temperature sensitivity of the maximum emission (590 $\mathrm{nm}$ ).

The sensitivity of the emission of $\mathrm{SiO}_{2} \mathrm{NPs}$ doped with $\mathrm{Ru}(\text { phen })_{3}{ }^{2+}$ is shown in Figure 4.b by plotting of the maximum of the emission $(590 \mathrm{~nm})$ as a function of the temperature. In both cases, Shell NPs $0.46 \%$ and Core NPs the intensity of the emission linearly decreased by increasing the temperature of the aqueous solution. This is due to the fact that the increase of the temperature favours the non-radiative deactivation pathways of the luminescent probe, in particular internal conversion. During the internal conversion, the electronic energy is converted to the vibrational energy of the luminescent probe itself accounting for the commonly observed decrease in fluorescence/phosphorescence intensity with temperature rising [35].

Furthermore Shell NPs $0.46 \%$ showed higher sensitivity to the temperature with respect to Core NPs. In fact a decrease of $1.45 \%{ }^{\circ} \mathrm{C}^{-1}$ was observed in the emission of Shell NPs, comparable with the data reported in literature for $\mathrm{Ru}(\mathrm{byp})_{3}-\mathrm{Shell} \mathrm{SiO}_{2} \mathrm{NPs}$ [26], whereas a lowering of $0.37 \%$ ${ }^{\circ} \mathrm{C}^{-1}$ was recorded in the case of Core NPs. The dye-SiO${ }_{2}$ architectures dramatically affect the optical properties of the luminophore due to different bonding and spatial hindrance. Studies have shown that doped core NPs present a considerable excimeric emission, whereas a monomeric emission is usually observed in the shell leading different responses of the emission to external factors [36]. Finally, it is obvious that luminophore immobilized on the external shell are more prone to variations of temperature since they are more exposed to the water. On the other hand, the $\mathrm{Ru}$ (phen $)_{3}{ }^{2+}$ immobilized in the core of NPs is less prone to temperature changes of aqueous solution due to the low thermal conductivity of the silica.

Figure 5 shows the maximum of the emission, which corresponds to 590nm, for Shell NPs, prepared with different loadings (wt\%) of the Ru complex (Ru(phen) ${ }_{3}{ }^{2+}$ ) respect to the silica in the shell. The emission from the nanoparticles increased as the amount of the luminescent probe increases till $1.2 \mathrm{wt} \%$ (which corresponds to Shell NPs $0.036 \%$, see Table 1 ). No Ru(phen) ${ }^{2+}$ could 
be detected in supernatant solution after the synthesis. On the contrary, for values above 1.2 wt.\% the intensity of the emission decreased. This is due to the fact that increasing the amount of the probe the formation of non-emissive aggregates is favored. Moreover, luminescent activity was noticed in the supernatant solution evidencing that $\mathrm{Ru}$ (phen $)_{3}{ }^{2+}$ was partially immobilized in the silica matrices due to the limited number of sides of interaction and the relatively fast growth of the shell. In the following experiments the load in all the Shell NPs was fixed to $1.2 \mathrm{wt} \%$. respect to the mass of $\mathrm{SiO}_{2}$ used to synthesize the shell.

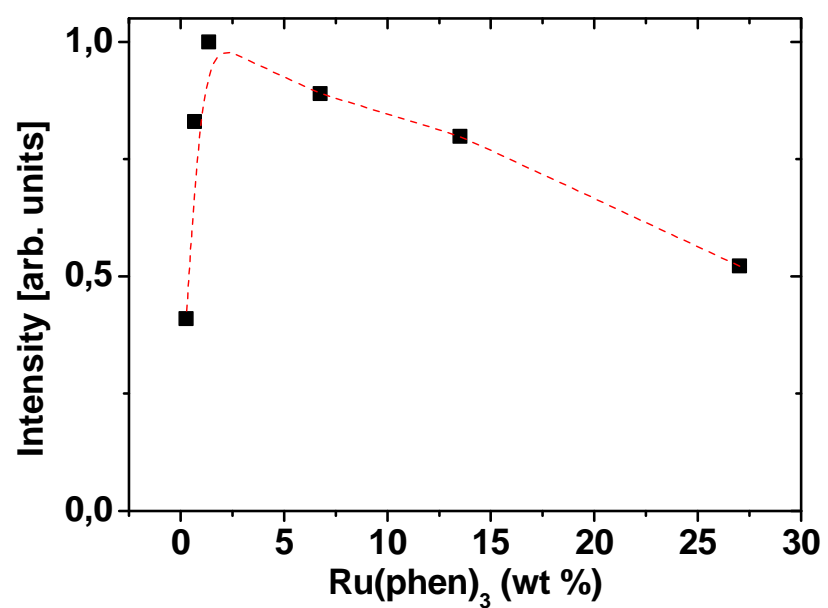

Figure 5. Normalized Intensity at $590 \mathrm{~nm}$ of Shell NPs prepared with different wt\%. of Ru(phen) ${ }_{3}{ }^{2+}$ respect to the silica in the shell $(0.027 \mathrm{~mL}$ of TEOS).

Shell NPs showed more promising results as nano-thermometer on the basis of their uniform shape combined with high emission and sensitivity towards the temperature. As reported in Table 1, different amount of TEOS were employed for the preparation of the shell made of silica in order to tune the properties of the developed nano-thermometer. Results in terms of Shell NPs diameter and shell thickness are reported in Figure 6. 


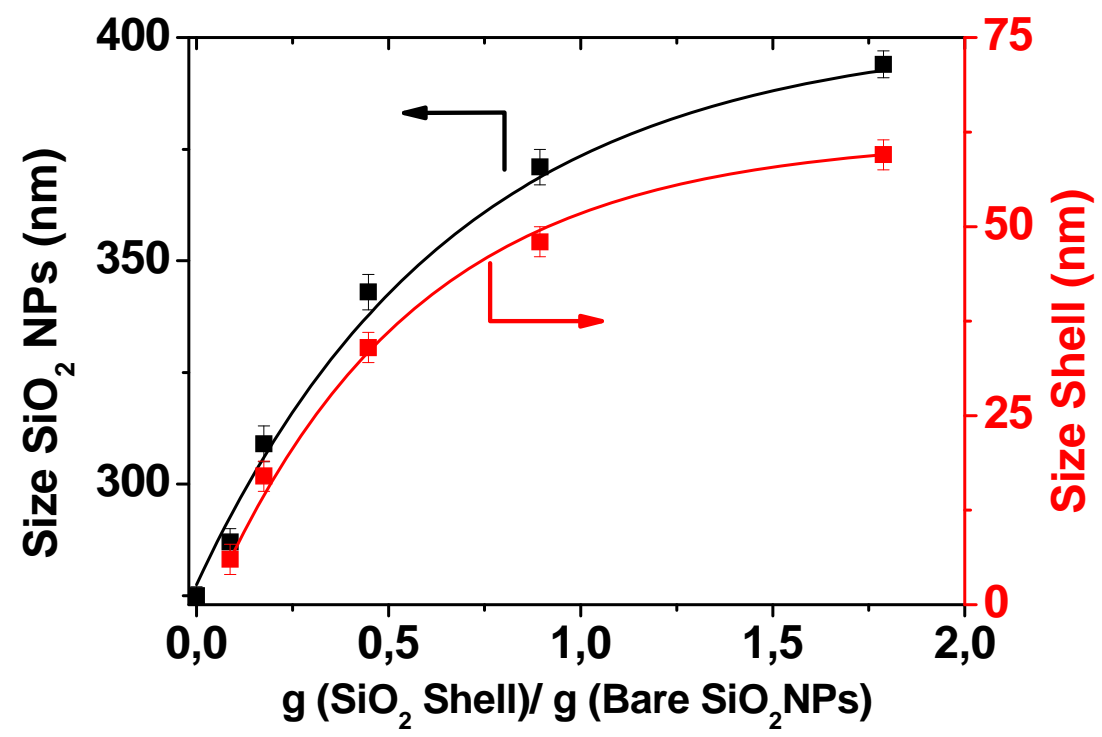

Figure 6. Particle size (black) and shell thickness (red) as a function of the mass ratio of silica precursor employed to grow the shell and the mass of Bare NPs coated. The values correspond to DLS measurements.

Measurements of the size of the NPs confirmed the presence of the external shell. In fact the diameter of the of the $\mathrm{SiO}_{2}$ NPs exponentially increased from $275 \mathrm{~nm}$ observed for Bare NPs to 394 $\mathrm{nm}$ for Shell NPs $0.46 \%$ as a function of the amount of $\mathrm{SiO}_{2}$ employed to synthesize the shell. This is due to the fact that the thickness of the shell exponentially increased by increasing the silica precursor employed during the preparation, as expected, from $3 \mathrm{~nm}$ (Shell NPs $0.036 \%$ ) to $57 \mathrm{~nm}$ (Shell NPs $0.46 \%$ ). On the other hand, the growth of the shell did not affect the narrow distribution of the nanoparticles, (see error bar in Figure 6) and the polydispersity of the nanoparticles is in all the cases around 0.05 .

From the emission spectra reported in Figure 7.a, it is possible to notice that the intensity of the emission increase by increasing the total amount of luminescent probe immobilized in the shell. In particular, the intensity of the maximum of emission (590 nm) linearly increased by increasing the molar concentration of $\mathrm{Ru}(\mathrm{phen})_{3}{ }^{2+}$ immobilized in the Shell NPs. This is due to the fact that the total amount of the luminophore in the aqueous solution containing the NPs increases as the shell size increases and the intensity of the emission, I, linearly increases with the concentration of the luminescent molecule (c) as predicted by the following equation:

$$
\boldsymbol{I}=\boldsymbol{k} \boldsymbol{I}_{\text {exc }} \Phi[\varepsilon \boldsymbol{b} \boldsymbol{c}]
$$

where $I_{\text {exc }}$ is the intensity of the exciting light, $\varepsilon$ the molar extinction coefficient, and $b$ is the optical path of the cuvette $(1 \mathrm{~cm})$. This confirms the absence of additional interfering phenomena due to the aggregation of the probe since no deviation from linear trend of the intensity of the emission as a function of the mole of $\mathrm{Ru}(\mathrm{phen})_{3}{ }^{2+}$. 
a)

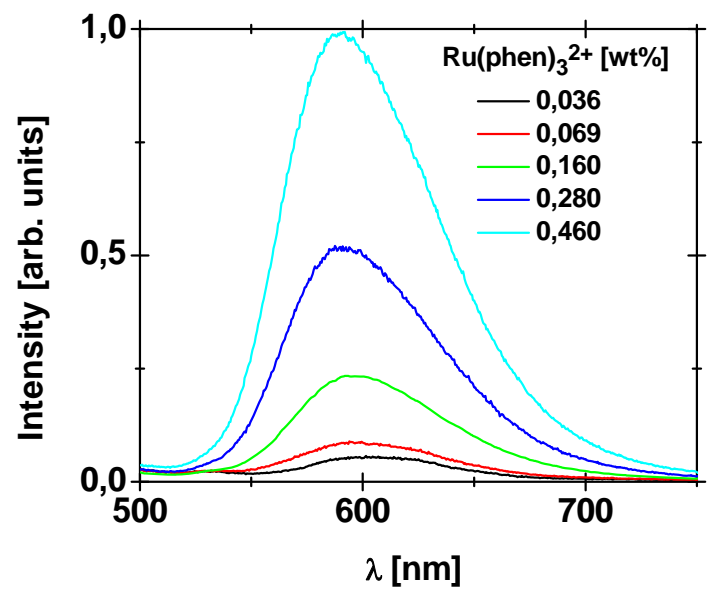

b)

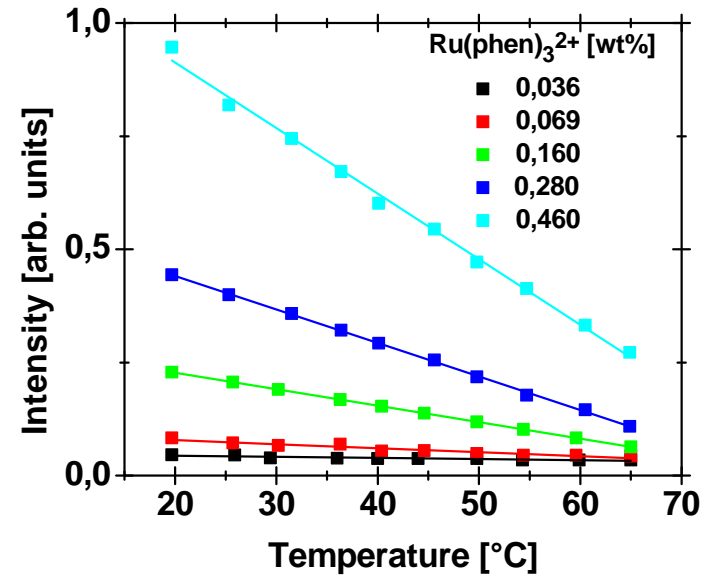

Figure 7. a) Normalized emission spectra of Shell NPs doped with different amounts of Ru(phen), b). Effect of the temperature on the intensity of the emission at $590 \mathrm{~nm}$ of Shell NPs doped with different amounts of $\mathrm{Ru}(\mathrm{phen})_{3}{ }^{2+}$

As shown in Figure 7.b, in all the cases, the intensity of the emission of the Ru Shell NPs linearly decreased $\left(\mathrm{R}^{2}>0.99\right)$ by increasing the temperature, from $20^{\circ} \mathrm{C}$ to $65^{\circ} \mathrm{C}$ which is the range of interest for membrane distillation processes. The increase of amount of $\mathrm{Ru}(\mathrm{phen})_{3}{ }^{2+}$ in the nanothermometer could promote higher degree of energy transfer between $\mathrm{Ru}(\mathrm{phen}){ }_{3}{ }^{2+}$ molecules, which results in a stronger emission quenching, producing a higher sensitivity towards temperature changes by increasing the number of luminescent probes immobilized in the shell. Moreover, the intensities reported in Figure 7.b refer to the mean values observed testing 3 different samples (2 cycles). The experimental error was below $2 \%$ in all the cases.

\subsection{Fluorescent Shell NPs immobilized in the surface of membranes as nanothermometers}

On the basis of the higher intensity of the emission and higher temperature sensitivity, the Shell NPs $0.46 \%$ were selected as the most promising candidate as nano-thermometer for monitoring the temperature on the hydrophobic PVDF membrane surface. This step is important in order to not affect the hydrophobicity of membrane surface required in such membrane processes (i.e. membrane distillation). The nanoparticles were functionalized with dodecyltriethoxysilane obtaining hydrophobic NPs made of silica stable in toluene solution, see Figure 8. We tried to evaluate the Z-potential of the colloidal hydrophobic NPs in toluene using the Phase Analysis Light Scattering (PALS) technique for the measurement of very small electrophoretic mobilities in nonpolar media [37]. Unfortunately, after several measurements the statistics were poor, mobilities varied from -0.072 to 0.136 and the measurement was discarded. The hydrophobic NPs were stable in ethanol (see the cuvette in Figure 9) and Z-potential measurement was successful resulting in a value of $-44.12 \pm 1.46 \mathrm{mV}$. 


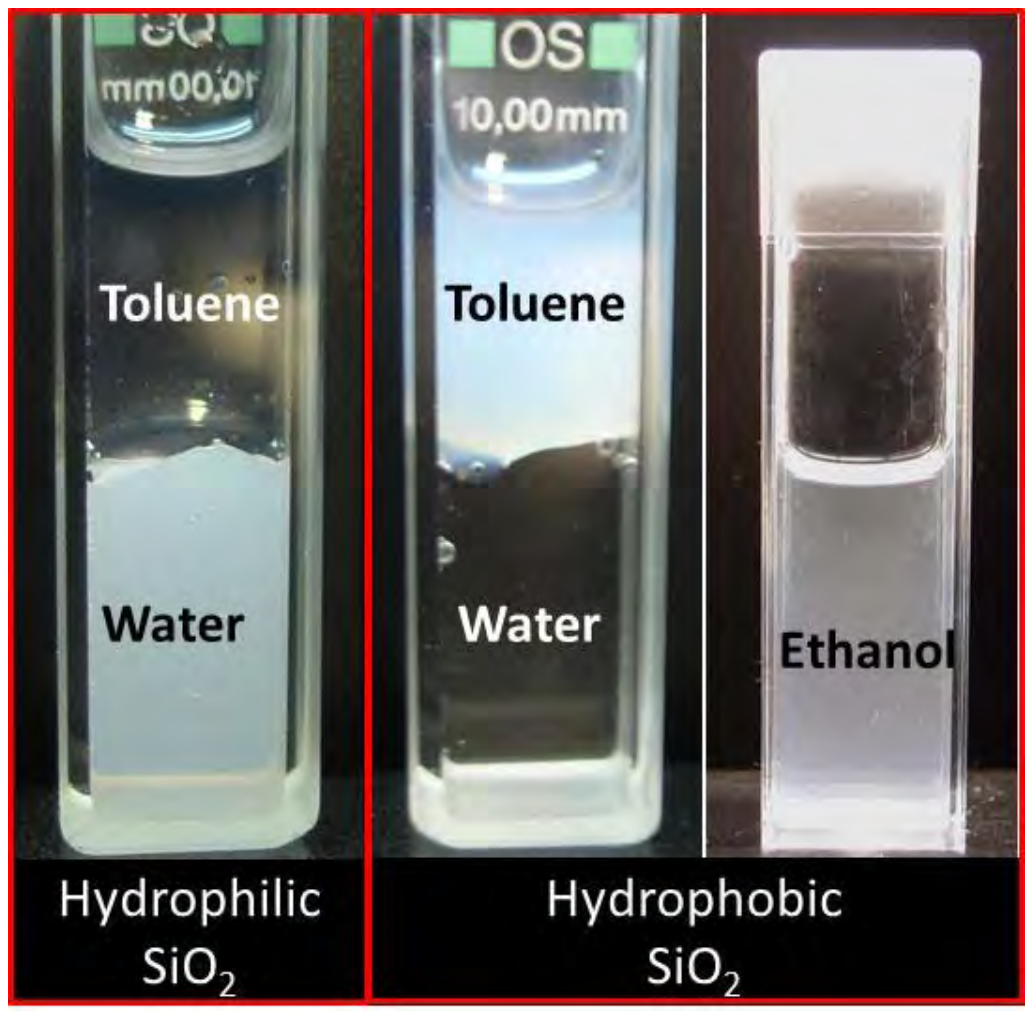

Figure 8. Images of hydrophilic and hydrophobic colloidal suspension of Shell NPs in different solvents.

In Figure 9 are depicted the cross-section micrographs of PVDF membrane load with Shell NPs $0.46 \%$ via dip-coating. PVDF membrane of ca $106 \mu \mathrm{m}$ of thickness presented a finger-like structure of ca $50 \mu \mathrm{m}$ supported on a spherulitical-like layer as reported previously [27]. A detailed analysis of the cross section, (Figure 9.b) and top view ( Figure 9.c), evidenced that the nanothermometer NPs were immobilized on membrane surface

a)

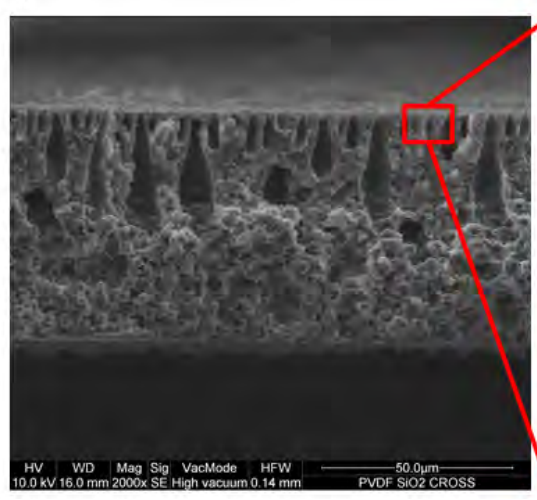

b)

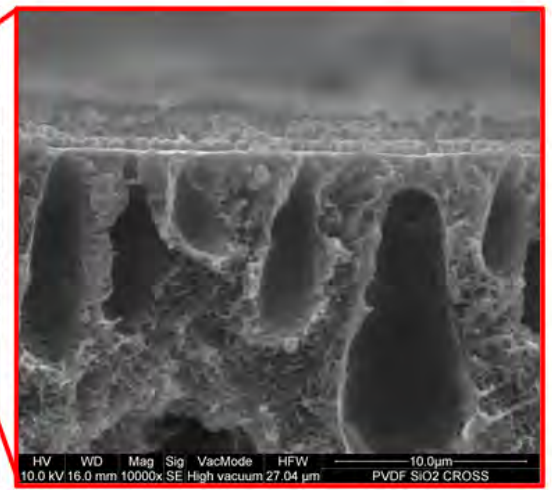

c)

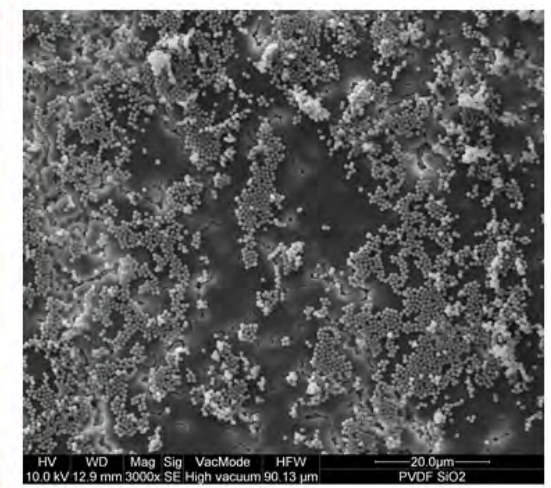

Figure 9. SEM picture of PVDF membrane load with hydrophobic $0.460 \%$ Shell NPs NPs: a) cross-section, b)zoom of the cross-section in proximity of the surface, c) top view of the surface.

By comparing the characterization of bare PVDF with PVDF load with Shell NPs membranes it is possible to conclude that the immobilization of the nano-thermometer did not affect 
the morphology of the membrane. In fact the contact angle is $109 \pm 2^{\circ}$ in the case of bare PVDF and $114 \pm 3^{\circ}$ in the case of PVDF loaded with functionalized $\mathrm{SiO}_{2} \mathrm{NPs}$. The increase of contact angle is due to the hydrophobicity of the functionalized $\mathrm{SiO}_{2} \mathrm{NPs}$ doped with $\mathrm{Ru}(\mathrm{phen})_{3}{ }^{2+}$ and the increase of the roughness of membrane surface as a consequence of the immobilization of the probes. In both cases the membrane porosity is in the range of $88 \pm 3 \%$ and LEP of $2.0 \pm 0.2$ bar, confirming that PVDF membranes preserved their morphology and integrity after immobilization of the nanothermometer.

Furthermore upon membrane excitation, (see Figure 9) it was observed an emission peak unmistakable attributed to the nano-thermometer doped with $\mathrm{Ru}(\mathrm{phen})_{3}{ }^{2+}$. The maximum of emission was observed at $614 \mathrm{~nm}$, red-shifted of $24 \mathrm{~nm}$ with respect to the emission observed for doped $\mathrm{Ru}(\text { phen })_{3}{ }^{2+}$ NPs dispersed in water. The red shift in the peak can be attributed to the higher refractive index of PVDF surface and coupling effect due to the higher packing density of nanoparticles on the surface and large change in polarity and polarizability of the fluorophore environment [38]. Furthermore, the larger widths on the surface are most likely due to inhomogeneous broadening resulting from the presence of a number of physically distinct environments at the attachment sites on the surface, whereas the decreasing in terms is attributed to the limited amount of doped $\mathrm{SiO}_{2}$ NPs immobilized on membrane surface. This configuration leads to higher sensitivity $\left(\mathrm{dI} / \mathrm{dT}=0.0143{ }^{\circ} \mathrm{C}^{-1}\right)$ towards the temperature compared to membrane prepared by immobilizing the luminescent probe in dense film made of PS $\left(\mathrm{dI} / \mathrm{dT}=0.0033^{\circ} \mathrm{C}^{-1}\right)$ [39]. On the other hand the temperature sensitivity of the fluorescent nano-particles is not affected by the immobilization on PVDF membranes as depicted in Figure 9.b. Furthermore, the immobilization of Shell NPs on membrane surface leaves the nano-thermometer in intimate contact with the environment and as a consequence is more prone to temperature variation. Furthermore, the immobilization in silica allows the protection of the probe from interfering agent (i.e. oxygen) and limits the photo-bleaching. Luminescent transition metal polypyridyl complexes suffer from pronounced cross talk to oxygen since their triplet states are subjected to severe collision quenching. However as reported in literature [13-14, 26], the dissolved oxygen has a null effect on the intensity of the emission of metal polypyridyl complexes such as $\mathrm{Ru}(\mathrm{phen})_{3}{ }^{2+}$ and $\mathrm{Ru}(\mathrm{byp}) 3^{2+}$ immobilized in silica due to the low oxygen permeability of the silica itself. 
a)

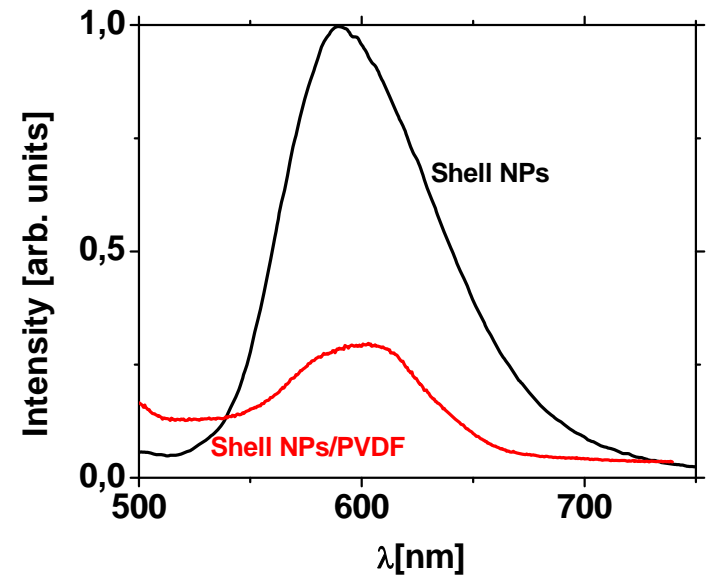

b)

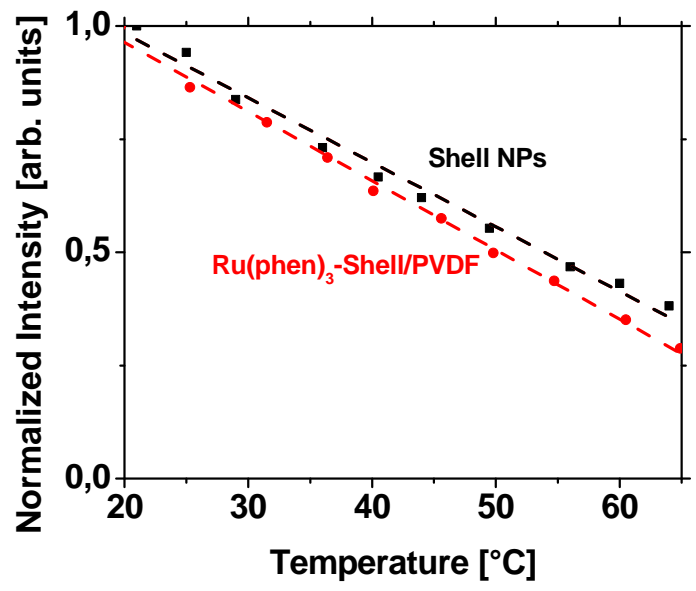

Figure 9. (a) Emission of Shell NPs immobilized on PVDF membranes compared with Emission of Shell NPs dispersed in water and (b) their temperature sensitivity.

To test the mechanical stability of the NPs attached to the surface, two membrane samples of area 2x2 cm were immersed in $50 \mathrm{ml}$ of water and subjected to sonication for $10 \mathrm{~min}$, after that the membranes were dried and the fluorescence was measured again. The immobilization of the nanoparticles is reasonable stable and only a decrease of $8 \%$ in the intensity of the fluorescence was observed, due to a minimal leak of the Shell NPs $0.46 \%$. It must be considered that sonication is a severe method to evaluate the stability of the coating. In fact, the immersion of the samples for 24 hours in water did not compromise the photochemical properties of the membrane since no variation of the intensity of the emission was observed. These experiments confirm the stability of the NPs immobilized on the surface of PVDF membranes.

$\mathrm{SiO}_{2}$ NPs are one of the most widely used inorganic materials for the fabrication of $\mathrm{PVDF} / \mathrm{SiO}_{2}$ composite membranes via blending method to enhanced hydrophilic properties of the membrane reducing membrane fouling. In general, $\mathrm{SiO}_{2} \mathrm{NPs}$ nanoparticles can be modified to form hybrid nanomaterials by chemically bonding or physically adsorbing polymers on silica surface to chemically anchor polymer chains on surface via "grafting to" or "grafting from". This technique could be of interest in facilitating the immobilization of $\mathrm{SiO}_{2} \mathrm{NPs}$ on polymeric surfaces improving the stability the nano-thermometer on membrane surface.

\section{Conclusions}

A nano-thermometer based on silica nanoparticles doped with Tris(1,10phenanthroline)ruthenium(II) $\left(\mathrm{Ru}(\mathrm{phen})_{3}{ }^{2+}\right.$ ) was prepared. The immobilization of the luminophore in an external shell made of silica consented to prepare monodispersed nanoparticles. On the other 
hand the immobilization of the luminescent molecule in the core, made of silica, dramatically affect the polydispersity of the NPs. As expected, the thickness of the shell was affected by the concentration of the silica precursor, whereas the mono-dispersity was maintained. The produced nanoparticles showed high photochemical activity and a strong phosphorescence ascribable to $\mathrm{Ru}$ (phen $)_{3}{ }^{2+}$. The intensity of the emission is modulated by the probe concentration. This systematic study contributed to the development of thermo-responsive colloids exploring the effect of the synthesis on photo-physical properties of the thermo-responsive nanoparticles and it showed that, on the basis of the concentration of the chemicals employed for the preparation of the Shell NPs it is possible to prepare monodispersed nano-thermometer with controlled and tunable properties in terms of particle size, luminescent activity and temperature sensitivity.

Furthermore, the intensity of the emission of the nanoparticles decreased linearly by increasing the temperature and is affected by the probe concentration. These results suggest the employment of the developed system as nano-thermometer offering the possibility of the imaging and mapping of the temperature in a wide range of applications (i.e. bio-medical).

The luminescent nanoparticles were immobilized on PVDF membrane maintaining their temperature sensitivity and providing information about the temperature on membrane surface. The coating was stable since the emission of the membrane remains constant after immersion of the membrane for $24 \mathrm{~h}$ in water and decreased by $8 \%$ after sonication for 10 minutes.

The impact of the deposition of the produced nanoparticles on the surface of the hydrophobic polymeric membrane opens a new perspective for non-invasive, on-line and in-situ monitoring and the mapping of temperature on membrane surfaces in real membrane processes. In particular, the immobilization of the developed fluorescent thermo-responsive nanoparticles on the surface of porous PVDF hydrophobic membrane by dip-coating could enable the evaluation of the thermal polarization in membrane distillation process.

\section{Acknowledgements}

Sergio Santoro would like to thank The Education, Audiovisual and Culture Executive Agency (EACEA) for the PhD grant under the Program "Erasmus Mundus Doctorate in Membrane Engineering” - EUDIME (http://www.eudime.unical.it). Artur Moro and Carla A. M. Portugal acknowledge the financial support of "Fundação para a Ciência e Tecnologia” (FCT-MCTES, Portugal) through the Post-Doc grants nr. SFRH / BPD / 69210 / 2010 and SFRH / BPD / 103619 /

2014. The People Program (CIG-Marie Curie Actions, REA grant agreement no. 321642), the Government of Aragon and the European Social Fund are gratefully acknowledged. CIBER-BBN is 
an initiative funded by the VI National R\&D\&i Plan 2008-2011 financed by the Instituto de Salud Carlos III with the assistance of the European Regional Development Fund.

The microscopy works have been conducted in the "Laboratorio de Microscopias Avanzadas" at "Instituto de Nanociencia de Aragon - Universidad de Zaragoza" . Authors acknowledge the LMAINA for offering access to their instruments and expertise. 


\section{References:}

[1] Vetrone, F, Naccache, R, Zamarrón, A, De La Fuente, A J, Sanz-Rodríguez, F, Maestro, L M,Rodriguez, E M, Jaque, D,Sole, J G, and Capobianco, J A. Temperature sensing using fluorescent nanothermometer. ACS Nano, 4(6): 3254-3258, 2010.

[2] Yang, J M, Yang, H, and Lin, L. Quantum dot nano thermometers reveal heterogeneous local thermogenesis in living cells. ACS Nano, 5(6): 5067-5071, 2001.

[3] Ross, D, Gaitan, M, and Locascio, L E. Temperature Measurement in Microfluidic Systems Using a Temperature-Dependent Fluorescent Dye. Anal. Chem., 73(17): 4117-4123, 2001.

[4] Filevich, O, and Etchenique, R. 1D and 2D Temperature Imaging with a Fluorescent Ruthenium Complex. Anal. Chem.,78(21): 7499-7503, 2006.

[5] Thamdrup, L H, Larsen, N B, and Kristensen, A. Light-induced local heating for thermophoretic manipulation of DNA in polymer micro- and nanochannels. Nano Lett.,10(3): 826-832, 2010.

[6] Wang, S, Westcott, S, and Chen, W. Nanoparticle Luminescence Thermometry. J. Phys. Chem. B, 106(43): 11203-11209, 2002.

[7] Wang, X D, Song, X H,He, C Y, Yang, C J, Chen, G, Chen, X. Preparation of Reversible Colorimetric Temperature Nanosensors and Their Application in Quantitative Two-Dimensional Thermo-Imaging. Anal. Chem., 83 (7): 2434-2437, 2011.

[8] Brites, C D S, Lima, P P, Silva, N J O, Milla, A, Amaral, V S, Palacio, F, and Carlos, L D. Thermometry at the nanoscale. Nanoscale, 4(16): 4799-4829, 2012.

[9] Santra, S, Zhang, P, Wang, K. M, Tapec, R, and Tan, W H. Conjugation of Biomolecules with Luminophore-Doped Silica Nanoparticles for Photostable Biomarkers. Anal. Chem., 73(20): 4988-4993, 2001.

[10] Canfarotta, F, Whitcombe, M J, and Piletsky, S A. Polymeric nanoparticles for optical sensing. Biotechnology Advances, 31(8): 1585-1599, 2013.

[11] Arduini, M, Marcuz, S, Montolli, M, Rampazzo, E, Mancin, F, Gross, S, Armelao, L, Tecilla, P, and Tonellato, U. Turning Fluorescent Dyes into Cu(II) Nanosensors. Langmuir, 21(20): 9314-9321, 2005.

[12] Stöber, W, Fink A, and Bohn, E. Controlled Growth of Monodisperse Silica Spheres in the Micron Size Range. J. Colloid Interface Sci., 26(1): 62-69, 1968.

[13] Bele, M, Siiman, O, and Matijevic', E. Preparation and Flow Cytometry of Uniform SilicaFluorescent Dye Microspheres. J. Colloid Interface Sci., 254(2): 274-282, 2002.

[14] Zhang, D, Wu, Z, Xu, J, Liang, J, Li, J, and Yang, W. Tuning the Emission Properties of Ru(phen)32+ Doped Silica Nanoparticles by Changing the Addition Time of the Dye during the Stöber Process. Langmuir, 26(9): 6657-6662, 2010. 
[15] Rossi, L M, Shi, L, Quina, F H, and Rosenzweig, Z. Stöber Synthesis of Monodispersed Luminescent Silica Nanoparticles for Bioanalytical Assays. Langmuir, 21(10): 4277-4280, 2005.

[16] Bagwe, R P, Yang, C, Hilliard, L R, and Tan, W. Optimization of Dye-Doped Silica Nanoparticles Prepared Using a Reverse Microemulsion Method. Langmuir, 20(19): 83368342, 2004.

[17] Qian, L, and Yang, X. One-Step Synthesis of Ru(2,2'-Bipyridine)3Cl2-Immobilized Silica Nanoparticles for Use in Electrogenerated Chemiluminescence Detection. Adv. Funct. Mater., 17(8): 1353-1358, 2007.

[18] Demas, J N, and DeGraff, B A. Applications of Luminescent Transition Platinum Group Metal Complexes to Sensor Technology and Molecular Probes. Coor. Chem Rev., 211: 317351, 2001.

[19] Liebsch, G, Klimant, I, and Wolfbeis, O S. Luminescence Lifetime Temperature Sensing Based on Sol-Gels and Poly(acrylonitrile)s Dyed with Ruthenium Metal-Ligand Complexes. Adv. Mater., 11(15): 1296-1299, 1999.

[20] Barja, B C, Chesta, C A, Atvars, T D Z, and Aramendía, P F. Fluorescent polymer coatings with tuneable sensitive range for remote temperature sensing. Spectrochimica Acta Part A: Molecular and Biomolecular Spectroscopy,116: 13-16, 2013.

[21] Liu, T, and Sullivan, J P. Physical Properties of Paints, in: Pressure and Temperature Sensitive Paints. Wiley, 2005.

[22] Alsaadi, A S, Francis, L, Amy, G L, and Ghaffour, N. Experimental and theoretical analyses of temperature polarization effect in vacuum membrane distillation. Journal of Membrane Science, 471: 138-148, 2014.

[23] Martínez-Díez, L, and Vázquez-González, M I. A method to evaluate coefficients affecting flux in membrane distillation. Journal of Membrane Science, 173(2): 225-234, 2000.

[24] Ali, A, Macedonio, F, Drioli, E, Aljlil, S, Alharbi, O A. Experimental and theoretical evaluation of temperature polarization phenomenon in direct contact membrane distillation. Chemical Engineering Research and Design, 91(10): 1966-1977, 2013.

[25] Tamburini, A, Pitò, P, Cipollina, A, Micale, G, and Ciofalo, M. A. Thermochromic Liquid Crystals Image Analysis technique to investigate temperature polarization in spacer-filled channels for Membrane Distillation. Journal of Membrane Science, 447: 260-273, 2013.

[26] Mirenda, M, Levi, V, Bossi, M L, Bruno, L, Bordoni, A, Regazzoni, A E, and Wolosiuk, A. Temperature response of luminescent tris(bipyridine)ruthenium(II)-doped silica nanoparticles. J. Colloid Interface Sci., 392: 96-101, 2013. 
[27] Drobek, M, Figoli, A, Santoro, S, Navascués, N, Motuzas, J, Simone, S, Algieri, C, Gaeta, N, Querze, L, Trotta, A, Barbieri, G, Mallada, R, Julbe, A, and Drioli, E. PVDF-mixed matrix membranes as VOCs adsorbers. Microporous and Mesoporous Materials, 207: 126-133, 2015.

[28] Brinker, C J, Frye, G C, Hurd, A J, Ashley, C S. Fundamentals of sol-gel dip coating. Thin Solid Films, 201: 97-108, 1991.

[29] Feng, C S, Shi, B, and Li, G. Preparation and properties of microporous membrane from poly (vinylidene fluoride-co-tetrafluoroethylene)(F2. 4) for membrane distillation. J. Membr. Sci., 237(1-2): 15-24, 2004.

[30] Drioli, E, Santoro, S, Simone, S, Barbieri, G, Brunetti, A, Macedonio, F, and Figoli, A. ECTFE membrane preparation for recovery of humidified gas streams using membrane condenser. Reactive \& Functional Polymers, 79: 1-7, 2015.

[31] Shibata, S, Taniguchi, T, Yano, T, and Yamane, M. Formation of Water-Soluble Dye-Doped Silica Particles. J. Sol-Gel Sci. Technol., 10(3): 263-268, 1997.

[32] Auger, A, Samuel, J, Poncelet, O, and Raccurt, O. A comparative study of non-covalent encapsulation methods for organic dyes into silica nanoparticles. Nanoscale Res Lett., 6: 328, 2011.

[33] Van Blaaderen, A, and Vrij, A. Synthesis and characterization of colloidal dispersions of fluorescent, monodisperse silica spheres. Langmuir, 8(12): 2921-2931, 1992.

[34] Zhang, D, Wu, Z, Xu, J, Liang, J, Li, J, and Yang, W. Tuning the emission properties of $\mathrm{Ru}($ phen)3(2+) doped silica nanoparticles by changing the addition time of the dye during the Stöber process. Langmuir, 26(9): 6657-6662, 2010.

[35] Sauer, M, Hofkens, J,and Enderlein, J. Basic Principles of Fluorescence Spectroscopy, in: Handbook of Fluorescence Spectroscopy and Imaging: From Single Molecules to Ensembles. Wiley, 2011.

[36] Rampazzo, E, Bonacchi, S, Montalti, M, Prodi, L, and Zaccheroni, N. Self-Organizing Core-Shell Nanostructures: Spontaneous Accumulation of Dye in the Core of Doped Silica Nanoparticles. J. Am. Chem. Soc, 129(46): 14251-14256, 2007.

[37] F. McNeil-Watson, W. Tscharnuter, J. Miller, A new instrument using phase analysis light scattering (PALS), Colloids Surf., A, 140,(1-3), 30: 53-57, 1998.

[38] McRae, E G. Theory of solvent effects on molecular electronic spectra. Frequency shifts. $J$. Phys. Chem. 61: 562-572, 1957.

[39] Santoro, S, Moro, A J, Portugal, C M, Crespo, J G, Coelhoso, I M, Lima, J C, 2016, Development of Oxygen and Temperature Sensitive Membranes Using Molecular Probes as Ratiometric Sensor. J. Membr. Sci., 514: 467-475, 2016. 